\title{
Aplicación de ensayos en discontinuo para la determinación de flujos de metano y dióxido de carbono en la degradación del material vegetal en el embalse Topocoro
}

\author{
Laura M. Lopera \\ lauralopera0614@gmail.co \\ $m$ \\ Lia M. Oviedo \\ liablue9215@hotmail.co \\ $\mathrm{m}$ \\ Gustavo A. Peñuela \\ gustavo.penuela@udea.edu. \\ co \\ Grupo Diagnóstico y Control de la Contaminación (GDCON), Escuela Ambiental, Facultad de Ingeniería, Sede de \\ Investigaciones Universitarias (SIU), Universidad de Antioquia (UdeA), Calle 70 No. 52-21, Medellín, Colombia
}

(Tipo de Artículo: Investigación Científica y Tecnología. Recibido el 02/08/2016. Aprobado el 22/09/2016)

\begin{abstract}
Resumen. La contribución de los embalses tropicales a las emisiones de gases de efecto invernadero (GEI) ha sido objeto de estudio recientemente, debido a los procesos de degradación y transformación del material vegetal que son llevados a cabo en estos, los cuales liberan metano $\left(\mathrm{CH}_{4}\right)$ y dióxido de carbono $\left(\mathrm{CO}_{2}\right)$, aportando al cambio climático. En el presente trabajo, se realizaron ensayos en discontinuo utilizando material vegetal de la zona de inundación del embalse Topocoro (Santander-Colombia), con el objetivo de determinar los flujos de $\mathrm{CH}_{4}$ y CO 2 de dicho material, simulando condiciones de inundación aerobias y anaerobias previas al llenado. Las mediciones de gases se realizaron empleando cromatografía de gases-masas (GC-MS). Las tasas de emisión bajo condiciones anaerobias variaron entre 118,513 - 377,666 Kg CH$/$ t.año y 120,870 - 553,758 Kg CO$/$ t.año, mientras que bajo condiciones aerobias las tasas de emisión fueron menores, con valores que oscilaron entre 0,73 y $8,83 \mathrm{Kg} \mathrm{CO}_{2} / \mathrm{t}$.año. Las emisiones producidas estuvieron determinadas principalmente por la cantidad de material vegetal inundado y por la presencia de organismos aerobios y anaerobios presentes en los sistemas en batch.
\end{abstract}

Palabras clave. Degradación; Dióxido de carbono; Embalses; Metano; Microorganismos.

\section{Batch tests application for the determination of flows of methane and carbon dioxide in the degradation of plant material in the reservoir Topocoro}

\begin{abstract}
The contribution of tropical reservoirs to emissions of greenhouse gases (GHGs) has been studied recently due to degradation processes and transformation of plant material are carried out in these, which release methane $\left(\mathrm{CH}_{4}\right)$ and dioxide carbon $\left(\mathrm{CO}_{2}\right)$, contributing to climate change. In this paper, tests were carried out in batch using plant material from the flood zone of Topocoro (Santander-Colombia) reservoir, in order to determine the flow of $\mathrm{CH}_{4}$ and $\mathrm{CO}_{2}$ of such material, simulating conditions of aerobic flood and previous anaerobic filling. Measurements were performed using gas chromatography-mass gas (GC-MS). The emission rates under anaerobic conditions varied between 118,513 - 377,666 Kg CH $4 /$ tyear y 120,870 - 553,758 Kg CO2/t.year, while under aerobic conditions the emission rates were less, with results that varied between 0,73 y 8,83 $\mathrm{Kg} \mathrm{CO} / \mathrm{t}$.year. The emissions produced were determinate mainly by the amount of flooded plant material and for the presence of aerobic and anaerobic organisms.
\end{abstract}

Keywords. Carbon dioxide; Degradation; Methane; Microorganisms; Reservoirs

\section{Introducción}

Los embalses para la generación de energía hidroeléctrica son sistemas acuáticos antropogénicos construidos en amplias extensiones geográficas, considerados de gran importancia para la producción de energía [1]. Tradicionalmente, la energía hidroeléctrica ha sido vista como una forma de energía limpia [2], sin embargo, actualmente ha sido puesto en duda debido a que se considera que en los embalses se pueden formar y liberar gases de efecto invernadero (GEI) como el dióxido de carbono y el metano [1].

Debido a la falta de consenso respecto al tema de la cuantificación de GEI que se liberan en los embalses, la comunidad hidroeléctrica internacional, a través de la Asociación Internacional de Hidroeléctricas (IHA) y el Programa Hidrológico Internacional de la Organización de las Naciones Unidas para la Educación, la Ciencia y la Cultura (UNESCO), realizaron las "Guías para la medición de GEI en Embalses de Agua Dulce" (GMGEIE). En ellas se orienta sobre la planeación y conducción de campañas de campo para estimar emisiones netas de GEl en embalses y se proponen métodos normalizados, de aplicación mundial, que aumentan la confiabilidad y facilitan la comparación de la información recolectada [3].

Las emisiones de GEI en un embalse se pueden atribuir a dos situaciones particulares: la primera durante el proceso de construcción en el cual se emplean combustibles fósiles en la maquinaria y la extracción de los materiales de construcción de la presa y cuarto de máquinas [4] y la segunda después de la inundación del embalse en el cual se da lugar a la descomposición de la materia orgánica sumergida dentro de los reservorios de agua [5]. Estas emisiones en los embalses pueden ser significativas dependiendo de características como la edad y la profundidad del embalse, la temperatura del agua, el área inundada, el uso del suelo antes de ser inundado el embalse, las prácticas de manejo, entre otras [6].

En este estudio, se realizó la estimación de las tasas de emisión de gases de efecto invernadero bajo condiciones anaerobias y aerobias por parte del material vegetal presente en la zona de inundación del embalse Topocoro, a través del desarrollo de ensayos en 
Ingenierías USBMed, Volumen 7, No. 2, Julio-Diciembre 2016

discontinuo, utilizando material vegetal recolectado en la zona antes del proceso de inundación y simulando las condiciones ambientales presentes en el embalse, tales como temperatura ambiental y concentración de nutrientes en el agua.

\section{Materiales y métodos}

\subsection{Zona de estudio}

El embalse Topocoro se localiza en el departamento de Santander, Colombia (Figura 1), en el cañón donde el río Sogamoso cruza la Serranía de La Paz, 75 km aguas arriba de su desembocadura en el río Magdalena y $62 \mathrm{~km}$ aguas abajo de la confluencia de los ríos Suárez y Chicamocha. La presa y el embalse se localizan en los municipios de Girón, Betulia, Zapatoca, Los Santos, Lebrija y San Vicente de Chucurí [7].

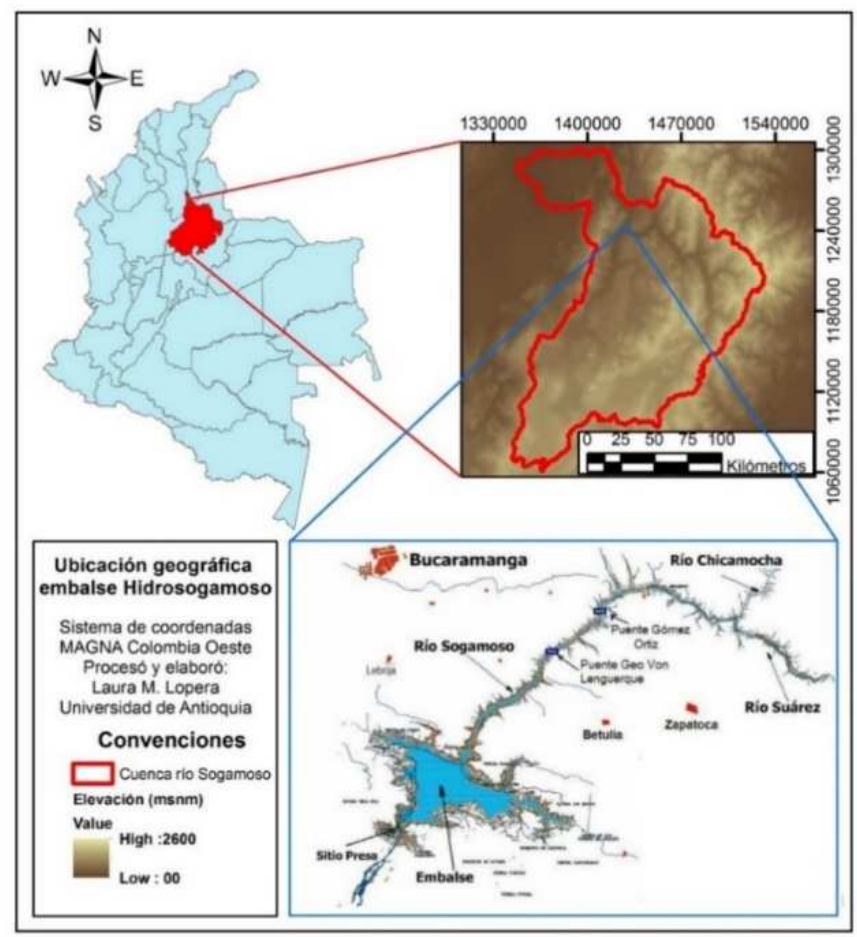

Figura 12. Zona de estudio, ubicación del embalse Topocoro

\subsection{Cobertura vegetal}

El área de inundación del embalse Topocoro se caracterizó por poseer diferentes tipos de cobertura vegetal, los cuales se presentan en la Tabla 2. Las coberturas más representativas por tamaño $e$ importancia fueron el bosque natural, los rastrojos altos y los pastizales arbolados.
Tabla 2. Cobertura vegetal. Modificado de Orrego et al. [8]

\begin{tabular}{ll}
\hline Tipo de cobertura & Área (ha) \\
\hline Bosque natural & 415,16 \\
\hline Pastizal arbolado & 2444,05 \\
\hline Rastrojo alto & 1719,58 \\
\hline Rastrojo bajo & 437,37 \\
\hline Rastrojo bajo xerofítico & 480,95 \\
\hline Pastizal & 623,28
\end{tabular}

El material vegetal en la zona de inundación del embalse se compone de árboles, raíces, hojarasca, hierbas y necromasa, los cuales contienen una cantidad aproximada de 295,32 kt de carbono.

\subsection{Recolección de muestras de material vegetal}

Se obtuvieron muestras de material vegetal en la zona de estudio en muestreos realizados entre los meses de julio y septiembre de 2014, correspondientes a diferentes especies, dentro de las cuales se pueden mencionar: Hyptis Sinuata, Cynodon, Ludwigia peruviana, Mimosa pigra, Pseudosamanea guachapele, Andropogon, Panicum, entre otras.

\subsection{Montaje experimental}

Se realizaron ensayos en discontinuo (durante 20 días) con el objetivo de determinar la cinética química de producción de los gases emitidos por el material vegetal, a fin de determinar la tasa de emisión para cada uno de los gases bajo diferentes condiciones. Para la incubación de las muestras se plantearon 12 experimentos en botellas de $160 \mathrm{ml}$, tanto para las condiciones aerobias como para las anaerobias, cuyo diseño de experimentos se muestra en la Tabla 2. Los ensayos fueron llevados a cabo por duplicado.

Tabla 3. Diseño de experimentos tanto para las condiciones aerobias como las anaerobias

\begin{tabular}{lllll}
\hline $\mathbf{N}^{\circ}$ & $\begin{array}{l}\text { Agua } \\
\text { sintética } \\
(\mathbf{m l})\end{array}$ & $\begin{array}{l}\text { Agua } \\
\text { embalse } \\
(\mathbf{m l})\end{array}$ & $\begin{array}{l}\text { Material } \\
\text { vegetal } \\
(\mathbf{g})\end{array}$ & $\begin{array}{l}\text { Lodo } \\
(\mathbf{m l})\end{array}$ \\
\hline $\mathbf{1}$ & 100 & 0 & 6 & 0 \\
\hline $\mathbf{2}$ & 100 & 0 & 6 & 10 \\
\hline $\mathbf{3}$ & 100 & 0 & 0 & 10 \\
\hline $\mathbf{4}$ & 0 & 100 & 6 & 0 \\
\hline $\mathbf{5}$ & 0 & 100 & 6 & 10 \\
\hline $\mathbf{6}$ & 0 & 100 & 0 & 10 \\
\hline
\end{tabular}

Para la mitad de los ensayos se empleó agua proveniente directamente del embalse y para la otra mitad se utilizó agua sintética preparada con las características del agua del embalse en cuanto a nutrientes y materia orgánica, la composición de esta fue: $23,4 \mathrm{mg} / \mathrm{L} \mathrm{C}_{6} \mathrm{H}_{12} \mathrm{O}_{6} ; 5,0 \mathrm{mg} / \mathrm{L} \mathrm{CH}_{4} \mathrm{~N}_{2} \mathrm{O} ; 0,1 \mathrm{mg} / \mathrm{L}$ $\mathrm{K}_{2} \mathrm{HPO}_{4}$. A cada botella se le adicionaron $100 \mathrm{ml}$ de agua dejando una cabeza libre de $60 \mathrm{ml}$ en cada botella para 
Ingenierías USBMed, Volumen 7, No. 2, Julio-Diciembre 2016

el almacenamiento de los gases producidos. Todas las botellas fueron selladas con tapones de butilo y sellos de aluminio. Para garantizar las condiciones anaerobias, las botellas se purgaron con nitrógeno gaseoso para eliminar el contenido de oxígeno. Por otro lado, para lograr las condiciones aerobias, se mantuvieron las botellas aireadas constantemente. Todos los ensayos fueron llevados a cabo a una temperatura controlada de $30^{\circ} \mathrm{C}$ simulando las condiciones de temperatura ambiente promedio en el embalse Topocoro. La tabla 3 muestra la caracterización del agua del embalse.

Tabla 4. Caracterización del agua del embalse

\begin{tabular}{llll}
\hline Parámetro & Promedio & máximo & mínimo \\
\hline $\mathrm{DBO}_{5}(\mathrm{mg} / \mathrm{L})$ & 5,97 & 22,00 & 5,00 \\
\hline $\mathrm{DQO}(\mathrm{mg} / \mathrm{L})$ & 26,72 & 64,19 & 25,00 \\
\hline $\mathrm{COD}(\mathrm{mg} / \mathrm{L})$ & 4,05 & 15,36 & 1,61 \\
\hline $\mathrm{CODB}(\mathrm{mg} / \mathrm{L})$ & 2,41 & 13,62 & 0,09 \\
\hline $\mathrm{ST}(\mathrm{mg} / \mathrm{L})$ & 1175,25 & 1696,00 & 122,00 \\
\hline $\mathrm{SST}(\mathrm{mg} / \mathrm{L})$ & 944,71 & 1400,00 & 10,00 \\
\hline $\mathrm{SD}(\mathrm{mg} / \mathrm{L})$ & 362,76 & 1390,00 & 46,00 \\
\hline $\mathrm{NH}{ }^{+}(\mathrm{mg} / \mathrm{L})$ & 5,02 & 5,70 & 5,00 \\
\hline $\mathrm{NTK}(\mathrm{mg} / \mathrm{L})$ & 5,06 & 7,31 & 5,00 \\
\hline $\mathrm{NO}_{3}{ }^{-}(\mathrm{mg} / \mathrm{L})$ & 1,37 & 4,46 & 0,21 \\
\hline $\mathrm{PT}(\mathrm{mg} / \mathrm{L})$ & 1,18 & 16,15 & 0,10 \\
\hline $\left.\mathrm{AT}^{(\mathrm{mg} \mathrm{CaCO}} / \mathrm{L}\right)$ & 70,80 & 143,40 & 32,30 \\
\hline $\mathrm{pH}$ & 7,89 & 8,65 & 6,02 \\
\hline
\end{tabular}

\subsection{Análisis de laboratorio}

Los ensayos fueron llevados a cabo en el laboratorio del grupo GDCON, laboratorio acreditado por el Instituto de Hidrología, Meteorología y Estudios Ambientales (IDEAM) en la norma 17025:2005 bajo la resolución 3564/2014. Se realizaron análisis de $\mathrm{pH}$, potencial redox (ORP) y oxígeno disuelto tanto para el agua del embalse como para el agua sintética, siguiendo las metodologías establecidas en el Estándar métodos [9, 10, 11].

\subsection{Mediciones de metano y dióxido de carbono}

Para las mediciones de $\mathrm{CO}_{2}$ y $\mathrm{CH}_{4}$ se utilizó un cromatógrafo de gases-masas (GC-MS) 7890A, con detector de masas 5975C y un detector de conductividad. Se seleccionó un volumen a inyectar de $0,4 \mathrm{ml}$. Para cada botella se obtuvo la muestra de la parte superior de la botella (columna de aire) usando una jeringa para la recolección de los gases y posteriormente se realizó inyección manual en el cromatógrafo.

\subsection{Curva de calibración}

Para la realización de la curva de calibración, se utilizaron bolsas Tedlar de 0,5 litros para hacer las diluciones de $\mathrm{CO}_{2}$ y $\mathrm{CH}_{4}$. En primer lugar, se llenaron 6 bolsas Tedlar con nitrógeno gaseoso y se adicionaron $0,5 \mathrm{ml} ; 1,0 \mathrm{ml} ; 5,0 \mathrm{ml} ; 10,0 \mathrm{ml} ; 30,0 \mathrm{ml}$ y $50,0 \mathrm{ml}$ tanto de
$\mathrm{CO}_{2}$ como de $\mathrm{CH}_{4}$ puro. Estos controles se midieron en el cromatógrafo GC-MS y se realizó la curva de área versus volumen, para obtener una ecuación para determinar el volumen de cada una de las mediciones.

\subsection{Determinación de las tasas de emisión}

Se realizaron gráficas de volumen versus tiempo para cada una de las muestras y se determinó la pendiente de cada gráfica $(\mathrm{ml} / \mathrm{d})$. Para expresar las tasas en unidades de mol/día se hizo uso de la ecuación de los gases ideales (Ecuación 1).

$$
\mathrm{PV}=\mathrm{nRT}(1)
$$

Donde:

$$
\mathrm{P}=\text { Presión ambiente }(0,84 \mathrm{~atm})
$$

$\mathrm{V}=$ Volumen emitido $(\mathrm{ml} / \mathrm{d})$

$\mathrm{n}=$ Número de moles $(\mathrm{mol} / \mathrm{d})$

$\mathrm{R}=$ Constante universal de los gases ideales $(0,082$ atm.L/mol.K)

$\mathrm{T}=$ temperatura ambiente $(303 \mathrm{~K})$

Finalmente las tasas fueron expresadas en términos de $\mathrm{Kg} \mathrm{CH}_{4}$ O $\mathrm{CO}_{2} /$ t.año.

\section{Análisis y resultados}

\subsection{Procesos anaerobios}

El pH inicial para el agua sintética y para el agua del embalse fue 6,635 y 8,878 , respectivamente, indicando condiciones de neutralidad en el agua sintética y condiciones un poco más básicas para el embalse. En el caso del potencial redox, ambos valores fueron positivos y muy cercanos; $251,3 \mathrm{mV}$ para el agua sintética y 260,4 $\mathrm{mV}$ para el agua del embalse, indicando condiciones oxidativas.

La Tabla 4 muestra los resultados obtenidos al finalizar los experimentos anaerobios en discontinuo. Los valores de $\mathrm{pH}$ se encontraron en el rango de 5,0 a 6,6 , siendo este último un pH óptimo para el desarrollo de bacterias metanogénicas [12]. La muestra 4 fue la que presentó el valor de $\mathrm{pH}$ más bajo, indicando formación de ácidos grasos por descomposición de la materia orgánica vía anaerobia, mientras que la muestra 2 presentó el valor de $\mathrm{pH}$ más alto, indicando baja actividad biológica, lo que sugiere falta de nutrientes 0 condiciones óptimas de las bacterias 0 arqueas anaerobias, para llevar a cabo la degradación. Todas las desviaciones estándar fueron bajas, con un promedio de 0,698 . 
Tabla 5. Parámetros al final del experimento anaerobio

\begin{tabular}{lll}
\hline Muestra & $\mathbf{p H}$ & $\begin{array}{l}\text { Potencial redox } \\
(\mathrm{mV})\end{array}$ \\
\hline $\mathbf{1}$ & 5,272 & $-55,5$ \\
\hline $\mathbf{2}$ & 6,466 & $-185,8$ \\
\hline $\mathbf{3}$ & 6,462 & $-161,7$ \\
\hline $\mathbf{4}$ & 5,032 & $-36,2$ \\
\hline $\mathbf{5}$ & 6,381 & $-200,8$ \\
\hline $\mathbf{6}$ & 6,638 & $-187,9$ \\
\hline
\end{tabular}

El potencial redox presentó valores negativos en todos los casos, indicando condiciones reductoras óptimas para el metabolismo de las arqueas metanogénicas [12, 13]. Las muestras que presentaron valores más altos de potencial redox fueron la 1 y la 4 , debido a que éstas sólo estaban compuestas por agua y material vegetal, lo que probablemente no aportaba una alta población de microorganismos metanogénicos, contrario a las otras muestras, a las cuales adicionalmente se les agregó una cantidad determinada de lodos anaerobios. El pH no presentó variaciones significativas entre las muestras, sin embargo fue más bajo que al iniciar el experimento.

\subsubsection{Tasas de emisión}

En la Tabla 5 se muestran las tasas de emisión obtenidas para metano y dióxido de carbono bajo condiciones anaerobias.

Tabla 5. Tasas de emisión bajo condiciones anaerobias

\begin{tabular}{|c|c|c|c|c|}
\hline Muestra & $\begin{array}{l}\text { Pendiente } \\
\text { (ml } \mathrm{CH}_{4} \\
\text { /día) }\end{array}$ & $\begin{array}{l}\text { Pendiente } \\
\text { (ml } \mathrm{CO}_{2} \\
\text { /día) }\end{array}$ & $\begin{array}{l}\text { Tasa } \\
\mathrm{Kg} \\
\mathrm{CH}_{4} / \text { t.año }\end{array}$ & $\begin{array}{l}\text { Tasa } \\
\mathrm{Kg} \\
\mathrm{CO}_{2} / \text { t.año }\end{array}$ \\
\hline 1 & N.D & 5,382 & N.D & 495,071 \\
\hline 2 & 10,673 & N.D & 357,819 & N.D \\
\hline 3 & 5,466 & 1,314 & 183,251 & 120,870 \\
\hline 4 & N.D & 6,020 & N.D & 553,758 \\
\hline 5 & 11,265 & N.D & 377,666 & N.D \\
\hline 6 & 3,535 & 1,341 & 118,513 & 123,354 \\
\hline
\end{tabular}

Las tasas halladas para cada uno de los compuestos son muy similares en las muestras que tenían la misma composición y sólo diferían en el tipo de agua utilizada. También se observó que las tasas más altas de emisión de metano se presentaron en las muestras 2 y 5 debido a que éstas contenían material vegetal y microorganismos formadores de metano. Por otro lado, las tasas más altas de producción de dióxido de carbono, se presentaron para las muestras 1 y 4 debido posiblemente a que no había presencia de microorganismos metanogénicos, pero sí de microorganismos acetogénicos, los cuales son capaces de transformar las moléculas simples a $\mathrm{CO}_{2}$.

La muestra 1 (material vegetal + agua sintética) no presentó emisiones significativas de metano, posiblemente debido a que no presentaba una cantidad importante de organismos metanogénicos, la mayoría de las emisiones estuvieron cerca de $0 \mathrm{ml}$, con un promedio de 0,175 $\mathrm{ml}$. Las emisiones de $\mathrm{CO}_{2}$ en el tiempo, presentaron una tendencia al aumento, con una pendiente positiva. Estas emisiones presentaron un promedio de $24,2 \mathrm{ml}$ y variaron entre un valor mínimo de $12,7 \mathrm{ml}$ y un valor máximo de $31,22 \mathrm{ml}$. El promedio de las emisiones de $\mathrm{CO}_{2}$ en esta muestra fueron de 495,071 $\mathrm{Kg} / \mathrm{t}$.año.

En la muestra 2 (material vegetal + lodos + agua sintética), las emisiones de metano aumentaron en el tiempo, presentando un valor mínimo de $10,137 \mathrm{ml}$ durante el primer día y un valor máximo de $33,490 \mathrm{ml}$. El promedio de las emisiones fue de $24,2 \mathrm{ml}$. A diferencia de las emisiones de metano, las emisiones de dióxido de carbono disminuyeron en el tiempo, presentando una pendiente negativa en la distribución de los datos. Durante las primeras 4 mediciones los datos aumentaron y disminuyeron de forma alterna, pero a partir de este día, las emisiones comenzaron a disminuir constantemente. El valor máximo fue $23,512 \mathrm{ml}$ en el día 15 y el valor mínimo fue $10,381 \mathrm{ml}$ durante el último día de mediciones. Las emisiones finales fueron $357,819 \mathrm{Kg}$ $\mathrm{CH}_{4} /$ t.año.

Para la muestra 3 (agua sintética + lodos), ambos gases se comportaron de una forma muy similar en el tiempo, mostrando una tendencia a aumentar a medida que pasaban los días. Para el caso de las emisiones de metano el promedio fue $14,918 \mathrm{ml}$, el valor máximo encontrado fue $17,933 \mathrm{ml}$ y el valor mínimo $6,534 \mathrm{ml}$, el cual se presentó como un dato atípico, ya que fue un valor numéricamente distante del resto. Por otro lado, las emisiones de dióxido de carbono presentaron un promedio de $3,259 \mathrm{ml}$, un valor máximo de $4,138 \mathrm{ml}$ y un mínimo de $1,188 \mathrm{ml}$ durante el primer día de mediciones, el cual también es considerado un dato atípico. En esta muestra fue posible cuantificar tanto las emisiones de metano como de dióxido de carbono, con valores del orden de $183,251 \mathrm{Kg} \mathrm{CH}_{4} / \mathrm{t}$.año y $120,870 \mathrm{Kg} \mathrm{CO}_{2} / \mathrm{t}$.año, respectivamente.

Las emisiones de metano y dióxido de carbono para la muestra 4 (material vegetal + agua embalse) tuvieron un comportamiento muy similar al presentado por la muestra 1. Eso es debido a que las muestras tenían la misma composición, a excepción del agua utilizada para cada una. Las emisiones de metano para la muestra 4 fueron muy poco significativas, la mayoría de las mediciones dieron como resultado $0 \mathrm{ml}$ y el valor máximo encontrado fue $0,548 \mathrm{ml}$. El promedio fue 0,138 $\mathrm{ml}$. Las emisiones de dióxido de carbono aumentaron a lo largo del tiempo, presentando un valor atípico mínimo de $12,464 \mathrm{ml}$ y un valor máximo de $25,718 \mathrm{ml}$ para el último día. La emisión de $\mathrm{CO}_{2}$ fue $553,758 \mathrm{Kg} \mathrm{CO}_{2} / \mathrm{t}$.año, la más alta de todas las monitoreadas.

Para la muestra 5 (material vegetal + lodo + agua embalse), en general las emisiones tuvieron una tendencia a aumentar en el tiempo al igual que en la 
muestra 2. El valor promedio de las emisiones fue $22,042 \mathrm{ml}$, oscilando entre un valor mínimo de 7,534 ml y un valor máximo de $27,898 \mathrm{ml}$. Para los datos de $\mathrm{CO}_{2}$ no se logró apreciar una tendencia determinada, debido a que inicialmente las concentraciones aumentaban y disminuían, pero durante las últimas 3 mediciones se observó que los valores disminuyeron constantemente. El valor máximo presentado fue $26,492 \mathrm{ml}$ y el mínimo $9,340 \mathrm{ml}$, con un promedio de $17,897 \mathrm{ml}$ y $377,666 \mathrm{Kg}$ $\mathrm{CH}_{4} / \mathrm{t}$.año.

En la muestra 6 (lodos + agua embalse), las emisiones de metano y dióxido de carbono presentaron un comportamiento similar respecto al tiempo al igual que como ocurrió con la muestra 3 de composición similar. Las emisiones de ambos gases presentan una tendencia constante a aumentar en el tiempo. Las emisiones encontradas fueron $118,513 \mathrm{Kg} \mathrm{CH}_{4} /$ taño y $123,354 \mathrm{Kg} \mathrm{CO} /$ t.año. Para el caso del metano, el promedio de emisiones fue $10,943 \mathrm{ml}$, con un valor máximo de $12,520 \mathrm{ml}$ en la última medición y un valor atípico mínimo de $4,773 \mathrm{ml}$. En el caso del dióxido de carbono, el promedio en las emisiones fue $2,621 \mathrm{ml}$, con un valor máximo de $3,406 \mathrm{ml}$ y un valor atípico mínimo de $0,687 \mathrm{ml}$.

Se determinó la correlación entre las emisiones de $\mathrm{CH}_{4}$ y CO 2 para cada una de las muestras (Tabla 6). Los coeficientes de correlación no fueron muy significativos, a excepción de las muestras 3 y 6 que presentaron coeficientes cercanos a 1 , indicando una relación directa entre las dos variables, es decir, si una aumenta o disminuye, la otra se comportará de la misma manera.

Tabla 6. Coeficientes de correlación entre metano y dióxido de carbono

\begin{tabular}{ll}
\hline Muestra & $\begin{array}{l}\text { Coeficiente de correlación } \\
\left(\mathrm{CH}_{4} \mathbf{y} \mathbf{C O}_{2}\right)\end{array}$ \\
\hline $\mathbf{1}$ & 0,491 \\
\hline $\mathbf{2}$ & $-0,149$ \\
\hline $\mathbf{3}$ & 0,996 \\
\hline $\mathbf{4}$ & 0,285 \\
\hline $\mathbf{6}$ & 0,217 \\
\hline
\end{tabular}

También se obtuvieron los coeficientes de correlación entre muestras de composición similar (Tabla 7). Los coeficientes de correlación son muy altos para todas las muestras tanto para las emisiones de $\mathrm{CO}_{2}$ como para las de $\mathrm{CH}_{4}$, mostrando una correlación positiva en todos los casos. Por lo tanto, el comportamiento de estas muestras fue muy similar, de este modo, cuando una aumentaba la otra también lo hacía y cuando una disminuía ocurría lo mismo con la otra. Esto demuestra que el tipo de agua no influyó en el comportamiento de las emisiones.

Las muestras que mejor representaron las condiciones reales bajo las cuales puede encontrarse el hipolimnio anaerobio de un embalse tropical fueron la 2 y la 5 ya que contenían material vegetal inundado y microorganismos anaerobios, los cuales son abundantes en los sedimentos. Para estas muestras, las emisiones de metano fueron mayores que las de dióxido de carbono, contrario a lo que se ha reportado en la literatura, donde las emisiones de $\mathrm{CO}_{2}$ siempre superan a las de $\mathrm{CH}_{4}$, sin embargo, esto se puede explicar debido a que en las botellas utilizadas el ambiente era completamente anaerobio, por lo cual el metano no tenía la posibilidad de oxidarse antes de ser emitido, como ocurre en condiciones reales.

El comportamiento de las emisiones tanto de metano como de dióxido de carbono durante el tiempo de duración del experimento anaerobio fueron muy similares para las muestras que tenían la misma composición y que sólo diferían en el tipo de agua, indicando que las emisiones estuvieron determinadas principalmente por la cantidad de material vegetal inundado y la presencia de organismos anaerobios. Por otro lado, las emisiones de metano fueron prácticamente nulas en las muestras en la cuales no fueron introducidos organismos anaerobios, lo cual es un indicador de que por sí solas, las plantas estudiadas no albergan cantidades significativas de organismos metanogénicos. Estas muestras en realidad no representan las condiciones que se presentarían en el hipolimnio anaerobio del embalse, sin embargo, puede considerarse como un indicador de las emisiones netas de las plantas.

En general, se observó que en la mayor parte de los casos, las emisiones tanto de metano como de dióxido de carbono bajo condiciones anaerobias aumentaron en el tiempo, lo cual puede indicar el comportamiento que tendrían estas emisiones en el embalse durante el tiempo inmediatamente posterior al llenado de este, sin embargo, para obtener información sobre la evolución de las emisiones a largo plazo es necesario llevar a cabo experimentos durante periodos de tiempo superiores al analizado en este caso.

Tabla 7. Coeficientes de correlación entre muestras de composición similar

\begin{tabular}{|c|c|c|}
\hline Muestras & $\begin{array}{ll}\text { Coeficiente } & \text { de } \\
\text { correlación }\left(\mathrm{CH}_{4}\right)\end{array}$ & $\begin{array}{l}\text { Coeficiente } \\
\text { correlación }\left(\mathrm{CO}_{2}\right)\end{array}$ \\
\hline 1 y 4 & 0,134 & 0,811 \\
\hline 2 y 5 & 0,873 & 0,797 \\
\hline 3 y 6 & 0,939 & 0,944 \\
\hline
\end{tabular}

\subsection{Procesos aerobios}

En la Tabla 8 se observan los resultados de oxígeno disuelto en aguas obtenidos para cada una de las muestras analizadas al final del experimento. Las botellas fueron aireadas al inicio con una concentración de oxígeno promedio de $6,75 \mathrm{mg} / \mathrm{L}$ y al final se cuantificó 
la concentración final (Tabla 8), corroborándose el consumo de éste por parte de los microrganismos.

Tabla 8. Oxígeno disuelto al finalizar el experimento aerobio

\begin{tabular}{cc}
\hline Muestra & $\begin{array}{c}\text { Oxígeno disuelto } \\
(\mathbf{m g} / \mathbf{L})\end{array}$ \\
\hline $\mathbf{1}$ & 0,9 \\
\hline $\mathbf{2}$ & 0,8 \\
\hline $\mathbf{3}$ & 0,5 \\
\hline $\mathbf{4}$ & 0,6 \\
\hline $\mathbf{5}$ & 0,7 \\
\hline $\mathbf{6}$ & 0,8
\end{tabular}

Se realizaron mediciones de $\mathrm{pH}$ para cada una de las muestras, al finalizar los experimentos. En la Tabla 9 se observa que si bien los resultados demuestran que hay una diminución en el $\mathrm{pH}$, por la formación de ácido carbónico, pero debido a que los volúmenes producidos de $\mathrm{CO}_{2}$ son bajos, los $\mathrm{pH}$ finales no se consideran ácidos, y se encuentran dentro de los valores apropiados en procesos biológicos, para el sostenimiento de la población microbiana. Es importante resaltar que el $\mathrm{CO}_{2}$ está asociado a las actividades de fotosíntesis, respiración, y junto con el calcio, se integra en el sistema carbonato, jugando un papel clave en la estabilización del pH del agua en los ecosistemas acuáticos [14].

Tabla 9. pH inicial y final del experimento aerobio

\begin{tabular}{|c|c|c|}
\hline Muestra & pH inicial & pH final \\
\hline 1 & \multirow{3}{*}{8,800} & 7,036 \\
\hline 2 & & 6,646 \\
\hline 3 & & 6,789 \\
\hline 4 & \multirow{3}{*}{6,600} & 7,010 \\
\hline 5 & & 7,220 \\
\hline 6 & & 7,310 \\
\hline
\end{tabular}

\subsubsection{Tasas de emisión}

Para todas las muestras se obtuvo un valor de pendiente bajo, esto se evidenció con los bajos volúmenes de $\mathrm{CO}_{2}$ medidos en el cromatógrafo. En la Tabla 10, se observa que las tasas de emisión expresadas en $\mathrm{Kg} / \mathrm{t}$.año, presentaron valores pequeños comparados con las emisiones obtenidas para las muestras bajo condiciones anaerobias, indicando que la cantidad de dióxido de carbono producido por cada tonelada de material vegetal en el embalse, en un año, no es lo suficientemente significativa, esto se puede explicar debido a que en el experimento la cantidad de oxígeno disuelto no era suficientemente significativa, para que continuara la degradación de la materia orgánica en la botella, y se logrará un valor de emisión más alto.
Tabla 10. Tasas de emisión $\mathrm{Kg} \mathrm{CO}_{2} / \mathrm{t}$ material vegetal al año

\begin{tabular}{ccc}
\hline Muestra & $\begin{array}{c}\text { Pendiente } \mathbf{C O}_{2} \\
\text { (ml/día) }\end{array}$ & $\begin{array}{c}\text { Tasa de emisión } \\
\text { (Kg/t.año) }\end{array}$ \\
\hline $\mathbf{1}$ & 0,96 & 8,83 \\
\hline $\mathbf{2}$ & 0,70 & 6,45 \\
\hline $\mathbf{3}$ & 0,14 & 1,28 \\
\hline $\mathbf{4}$ & 0,80 & 0,73 \\
\hline $\mathbf{5}$ & 0,89 & 8,22 \\
\hline $\mathbf{6}$ & 0,29 & 2,65
\end{tabular}

Las tasas de emisión de dióxido de carbono bajo condiciones aerobias, tuvieron valores bajos, indicando que la producción de $\mathrm{CO}_{2}$ por la degradación aerobia no es lo suficientemente significativa en el embalse, esto es debido a que muchas veces la tasa de fijación de $\mathrm{CO}_{2}$ supera la tasa de emisión del mismo y también a que hay mayor producción de $\mathrm{CO}_{2}$ proveniente de la degradación anaerobia. En el caso de las concentraciones de oxígeno disuelto, se obtuvieron valores muy bajos en el experimento, lo cual podría explicarse de como se trata de un sistema lentico el flujo de agua no es lo suficientemente significativo como para tener una aireación constante en el cuerpo de agua y tener niveles de concentración altos de oxígeno disuelto, sin embargo se considera que las concentraciones de oxígeno en la parte superior del embalse son mayores que los valores obtenido en el experimento, por lo tanto se hace necesario mejorar la adecuación de estos sistemas aerobios.

Los volúmenes de emisión fueron similares para las muestras con agua sintética y las muestras con agua del embalse, indicando que es factible realizar experimentos con agua preparada de acuerdo a las características del agua del embalse en casos en los que sea difícil llevar a cabo muestreos en una determinada zona.

\section{Conclusión}

La degradación de biomasa vegetal por procesos anaerobios ocasionó una mayor emisión de GEI, principalmente de metano, comparado con las condiciones simuladas en procesos aerobios. Estas condiciones son comunes en embalses tropicales, donde generalmente se presentan una disminución del oxígeno en la columna de agua, por lo cual son más propensos a emitir metano como principal gas de efecto invernadero.

\section{Agradecimientos}

A la empresa ISAGEN y el grupo GDCON de la Universidad de Antioquia por la financiación, mediante el convenio 47/26: "Cuantificación de emisiones de GEI para el Proyecto Hidroeléctrico Sogamoso". 


\section{Referencias}

[1] Ometto, J. P., Cimbleris, A. C. (2013). Carbon emission as a function of energy generation in hydroelectric reservoirs in Brazilian dry tropical biome. Energy policy, 109-116.

[2] Bambace, L., Ramos, F. (2007). Mitigation and recovery of methane emissions from tropical hydroelectric dams. Energy, 1038-1046.

[3] International Hydropower Association (IHA). (2010). GHG Measurement guidelines for freswater reservoirs. Derived from: The UNESCO/IHA Greenhouse Gas Emissions from freshwater Reservoirs Research project, 1 ed. London.

[4] Demarty, M., Bastien, J. (2011). GHG emissions from hydroelectric reservoirs in tropical and equatorial regions: Review of 20 years of $\mathrm{CH} 4$ emission measurements, Energy Policy, 39 (7): 41974206.

[5] Pinguelli Rosa, L., Schaeffer, R. (1995). Global warming potentials: The case of emissions from dams. Energy Policy, 149-158.

[6] Rengifo, O. D., Londoño, J. A., Orozco, M. L. (Abril, 2011). Estudio de metodologías para estimar emisiones de GEI por embalses. Medellín, Colombia.

[7] ISAGEN. (2014). Proyecto Hidroeléctrico Sogamoso. [1 Enero de 2014]. Disponible en: http://www.isagen.com.co/comunicados/CartillaSogamoso_2013 .pdf.
[8] Orrego, S.A., Del Valle, J.I., Moreno, F.H. (2002). Medición de la captura de carbono en ecosiste, forestales tropicales de Colombia. Contribución para la mitigación del cambio climático. Universidad Nacional de Colombia. Sede Medellín. Departamento de Ciencias Forestales. Primera edición. 309 p.

[9] APHA, AWWA, WPCF. (2012). Standard Methods for the Examination of Water and Wastewater, 21th ed. Washington, DC.

[10] Severiche, C., \& González, H. (2012). Evaluación analítica para la determinación de sulfatos en aguas por método turbidimétrico modificado. Revista Ingeniería USBMed, 3 (2), 6-11.

[11] Severiche, C., \& González, H. (2013). Verificación analítica para las determinaciones de cromo hexavalente en aguas por espectrofotometría. Revista Ingeniería USBMed, 4 (1), 22-26.

[12] Torres, R., Ramírez, F., Fernández, F., \& Barriga, I. (2005). Methanogenesis and methane oxidation in wetlands. Imlications in the global carbon cycle. Hidrobiológica, 327-349.

[13] Rivas, C., \& Mota, M. (2008). Instituto de Higiene, Universidad de la República de Uruguay. Obtenido de Bacterias anaerobias: http://www.uprm.edu/biology/profs/massol/manual/p2-redox.pdf

[14] Palau, A., Alonso, M., \& Corregidor, D. (2010). Análisis de ciclo del carbono en embalses y su posible efecto en el cambio climático. Aplicación al embalse del Susqueda (Río Ter, España). Ingeniería del agua, 247-255. 